\title{
Who Gets Testosterone? Patient Characteristics Associated with Testosterone Prescribing in the Veteran Affairs System: A Cross-Sectional Study
}

\author{
Guneet K. Jasuja, $P h D^{1,2}$ and Adam J. Rose, MD, MSc, FACP \\ 'Center for Healthcare Organization and Implementation Research (CHOIR), ENRM VAMC, Bedford VA Medical Center, Bedford, MA, USA; \\ ${ }^{2}$ Department of Health Law, Policy and Management, Boston University School of Public Health, Boston, MA, USA; ${ }^{3}$ Department of Medicine, \\ Section of General Internal Medicine, Boston University School of Medicine, Boston, MA, USA.
}

J Gen Intern Med 32(10): 1075

DOI: $10.1007 / \mathrm{s} 11606-017-4113-\mathrm{Z}$

(c) Society of General Internal Medicine (outside USA) 2017

I $\mathrm{n}$ our previous study, we examined whether initiation of testosterone was preceded by appropriate diagnostic evaluation in accordance with clinical guidelines. ${ }^{1}$ In contrast, the present study focused on which patient characteristics were associated with testosterone prescribing among those who did not have a classical indication for receiving it. ${ }^{2}$ Thus, these studies looked at two distinct, though related, issues of recommended workup and valid indication, and should not be conflated. It is noteworthy that while Dr. Jackson's seven patients would not have met the criteria in our other paper for completing the full workup prior to starting testosterone, they also would not have met criteria for no workup at all.

It is certainly true that International Classification of Diseases (ICD) diagnosis codes are not always accurately applied and recorded by practicing clinicians. ${ }^{3,4}$ Though these codes have played a significant role in advancing research and policymaking in general, underreporting of chronic conditions with these codes is common. ${ }^{3}$ As reported in the limitations section of our paper, we addressed this concern by requiring two ICD-9 codes for confirming the condition. It is also worth remembering that not all ICD codes are created equal in terms of under-coding. Hypogonadism may be under-coded, which is why we did not consider its absence as constituting inappropriate prescribing in the prior paper. However, it would be surprising if a condition as consequential as Klinefelter syndrome or pituitary adenoma were not accompanied by an ICD code. Therefore, while it may be shocking that $94 \%$ of testosterone prescribing in Veteran Affairs (VA) was off-label and possibly not indicated, that does not mean this finding is inaccurate. Our more important findings may be the predictors of testosterone receipt in men without a classical indication for receiving it — most notably obesity, opioid use, diabetes, and depression. This points to the possibility that testosterone is being given for reasons other than simple hypogonadism, at least in part.

Corresponding Author: Guneet K. Jasuja, PhD; Center for Healthcare Organization and Implementation Research (CHOIR)ENRM VAMC, Bedford VA Medical Center, 200 Springs Road, Bedford, MA 01730, USA (e-mail: guneet.jasuja@va.gov).

\section{Compliance with Ethical Standards:}

Conflict of Interest: Neither of the authors have any conflict of interest.

\section{REFERENCES}

1. Jasuja GK, Bhasin S, Reisman JI, Berlowitz DR, Rose AJ. Ascertainment of testosterone prescribing practices in the VA. Med Care. 2015;53(9):746-52.

2. Jasuja GK, Bhasin S, Reisman JI, et al. Who gets testosterone? Patient characteristics associated with testosterone prescribing in the Veteran Affairs system: a cross-sectional study. J Gen Intern Med. 2017;32(3):30411.

3. Garvin JH, Redd A, Bolton D, et al. Exploration of ICD-9-CM coding of chronic disease within the Elixhauser comorbidity measure in patients with chronic heart failure. Perspect Health Inf Manag. 2013;10(Fall): 1b

4. O'Malley KJ, Cook KF, Price MD, Wildes KR, Hurdle JF, Ashton CM. Measuring diagnoses: ICD code accuracy. Health Serv Res. 2005;40(5 Pt 2):1620-39. doi: 10.1111/j.1475-6773.2005.00444. 